\title{
Gender Differences: Mortgage Credit Experience
}

\author{
Debby Lindsey-Taliefero \\ School of Business, Howard University, Washington DC, USA \\ Email: dlindsey@howard.edu
}

Received 5 August 2015; accepted 15 September 2015; published 18 September 2015

Copyright (C) 2015 by author and Scientific Research Publishing Inc.

This work is licensed under the Creative Commons Attribution International License (CC BY). http://creativecommons.org/licenses/by/4.0/

(c) (i) Open Access

\section{Abstract}

This study examined gender differences in the mortgage credit experience. Home Mortgage Disclosure Data Act (HMDA) data along with Lending Patterns ${ }^{\mathrm{TM}}$ generated rates for originations, denials, and fallouts from 2004 to 2013 . The gender effect on these rates was examined for statistical differences using the independent t-test, ANOVA, and one sample t-test. Across the country, the results showed no statistical gender effect on origination, denial or fallout rates in the posthousing crisis era. Within the race, this relationship held up, with exclusions. The white females had a lower fallout rate than white males, and Asian females had a higher denial rate than Asian males. Within gender, controlling for race, white females had higher origination and lower denial rates than Black, Hispanic, and Native Americans but were not statistically different from Asian females. Comparing white males to females by race, the results indicated that $53 \%$ of the time white males had higher origination, lower denial, or lower fallout rates than females. While $40 \%$ of the time, the white male's mortgage experience was not statistically different from females. Seven percent of the time the white male's origination rate was lower than white females. In the final analysis, at no time did minority females have a better mortgage experience than white males, but they did $33 \%$ of the time experience no statistical difference. Given these points, the applicant's gender had less of an effect on the mortgage credit experience than the applicant's race.

\section{Keywords}

Gender, Mortgage, and Lending Disparities, Fair Lending, Equal Credit Opportunity

\section{Introduction}

The Economic Report of the President [1] details that the US economy experienced a breakthrough year in 2014. The US economy has added jobs at the fastest pace since the 1990s. The unemployment rate dipped to its lowest 
level in over six years. The housing market is rebounding according to market indicators including the MultiIndicator Market Index ${ }^{\circledR}\left(\mathrm{MiMi}^{\circledR}\right)$ [2], the National Housing Survey ${ }^{\mathrm{TM}}$ [3], and Mortgage Lender Sentiment Survey $^{\mathrm{TM}}$ [4]. In general, these indicators point to a strong demand for home purchase, a continued increase in home prices, and the gradual decline in credit tightening. With this in mind, does a healthy recovery in the mortgage market mean that borrowers will have an evenhanded shot at homeownership? Or, will there be gender differences in the experience of obtaining mortgages in the post-housing crisis milieu?

Gender disparities in lending have been a growing concern of policymakers and researchers. As a consequence, an increasing number of mortgage gender studies have come out over the past 15 years. These works range from describing differences in male versus female origination or denial rates (Hedderly [5] and National Council of Negro Women [6]) to incorporating a gender variable into models that capture the mortgage underwriting decision (O’Connor [7] and Robinson [8]). The samples in this body of literature are diverse but can be broadly grouped as regional (Woodstock Institute [9], Cyr [10], Mohanty [11] and [Diabate and Johannsson [12]) and national (Awoonor-William [13] and Dietrich [14]). Since the housing crisis, there has been a surge in gender subprime mortgage, (Jordain-Earl [15], Dymski [16] and Cheng, Lin and Liu [17]) and foreclosure studies (Phillips [18] and Baker [19]). On a whole, the findings from the mortgage gender studies are inconclusive. Frequently, studies that have found no gender issue either on approvals or denials used empirical models and national or regional data. On the other hand, studies that found a gender effect usually were regional used descriptive analysis or decision models but often had specialized samples. This study examined gender differences in the mortgage credit experience in the post-housing crisis milieu by testing for statistical differences in origination, denial, and fallout rates. This work adds to the mortgage gender literature by increasing the investigation period, testing fallouts, and assessing distinctive cohorts.

\section{Method}

This research uses 2004 to 2013 data from Lending Patterns ${ }^{\mathrm{TM}}$ [20], which is an online HMDA data analysis tool that delineates the lending disposition of most lenders in the US by race, income, and geography. In particular, Lending Patterns ${ }^{\mathrm{TM}}$ generates loan processing rates that measure lenders' behavior during the loan process. These rates included origination, denial, and fallout rates. These rates can reveal how well the lender manages their loan processing from taking an application to making a decision to approve or deny a mortgage. The origination rate is a percentage that measure the number of loans originated divided by the total number of loan applications. The denial rate is a percentage that measure the number of loans denied divided by the total number of loan applications. Fallout rate is a percentage that measures the number of loans not close for reasons other than denial divided by the total number of loan applications. Fallouts can include applications that are approved but rejected by the applicant, withdrawn by the applicant, or incomplete. Finally, to test the gender effect on origination, denial, and fallout rates a mixture of statistical procedures was used, including independent t-test, one-way analysis of variance, and one-sample t-test.

\section{Participants}

The participants are mortgage applicants for a loan to purchase or refinance a home that contained 1 to 4 units. The types of mortgage loans included are conventional, Federal Housing Administration (FHA) and Veterans Affairs (VA). The mortgage borrower(s) included male only, male with co-borrower male, female only, or female with co-borrower female. This delimitation was done to untainted the gender equality comparisons. Figure 1 and Figure 2 provide origination, denial, and fallout distributions of the applicants. Male applicants’ prehousing crisis annual averages for origination, denial and fallout rates are 51.65 (SD = 2.91), $23.42(\mathrm{SD}=3.38$ ), and 24.92 (SD = 0.53), respectively, while post housing crisis are 56.70 (SD = 4.71), 22.16 (SD = 3.48), and 21.15 (SD = 1.29), respectively. Female applicants’ pre-housing crisis annual averages for origination, denial and fallout rates are $51.97(\mathrm{SD}=3.13), 24.71(\mathrm{SD}=3.82)$, and 23.31 (SD = 0.86), respectively, while post housing crisis are 60.32 ( $\mathrm{SD}=1.59), 20.20(\mathrm{SD}=0.97)$, and 19.48 ( $\mathrm{SD}=0.78)$, respectively. The majority of the applicants, pre and post housing crisis, were males $58 \%$ vs. females $42 \%$ and $59 \%$ vs. females $42 \%$, respectively. The partitioning of the applicants' race consisted of whites, 69\% (of this 60\% male vs. 40\% female), Blacks $13 \%$ (of this $46 \%$ male vs. 54\% female), Hispanics 13\% (of this $62 \%$ male vs. 38\% female), and Asians $5 \%$ (of this $60 \%$ male vs. $40 \%$ female) and Native Americans $0.4 \%$ (of this $57 \%$ male vs. $43 \%$ female). 


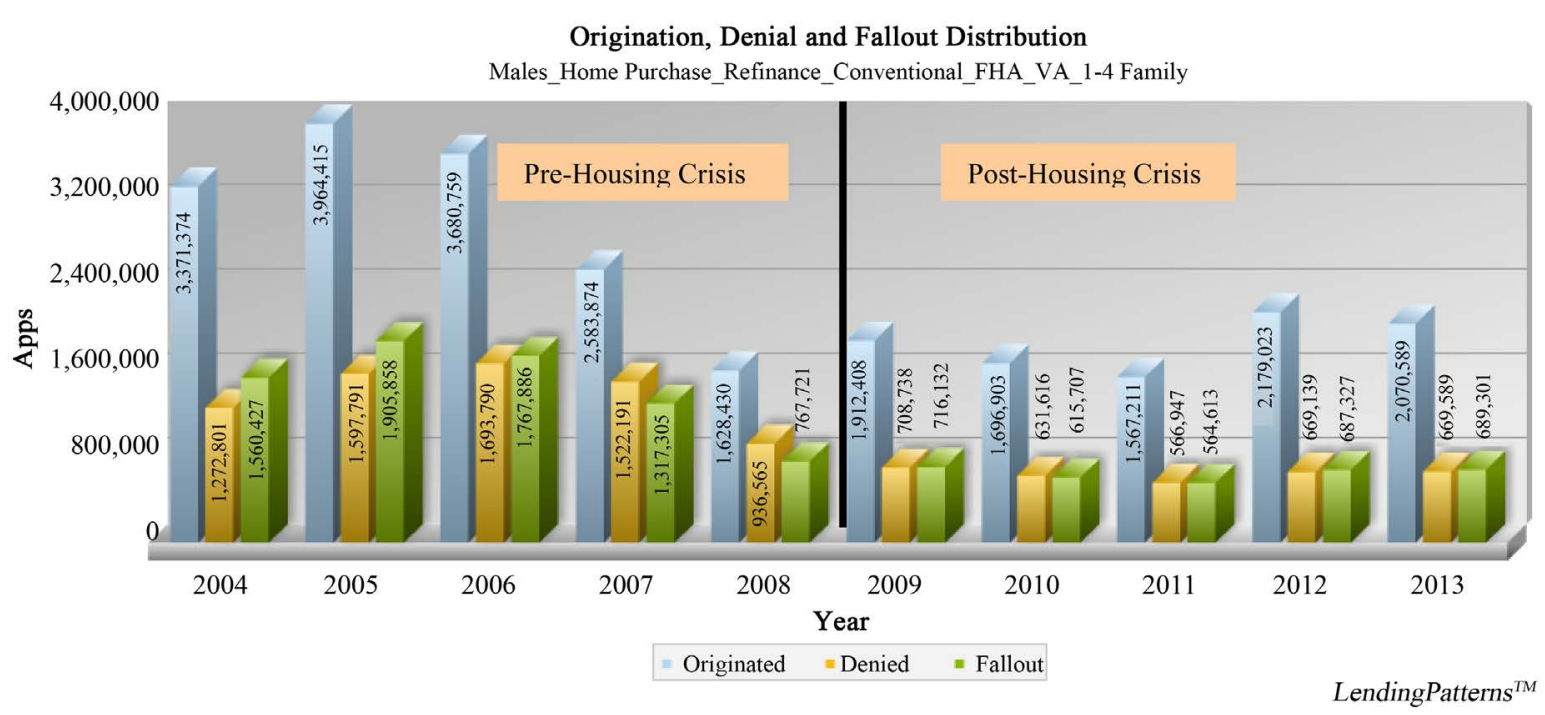

Figure 1. Male applicants: pre and post housing crisis origination, denial, fallout distribution.

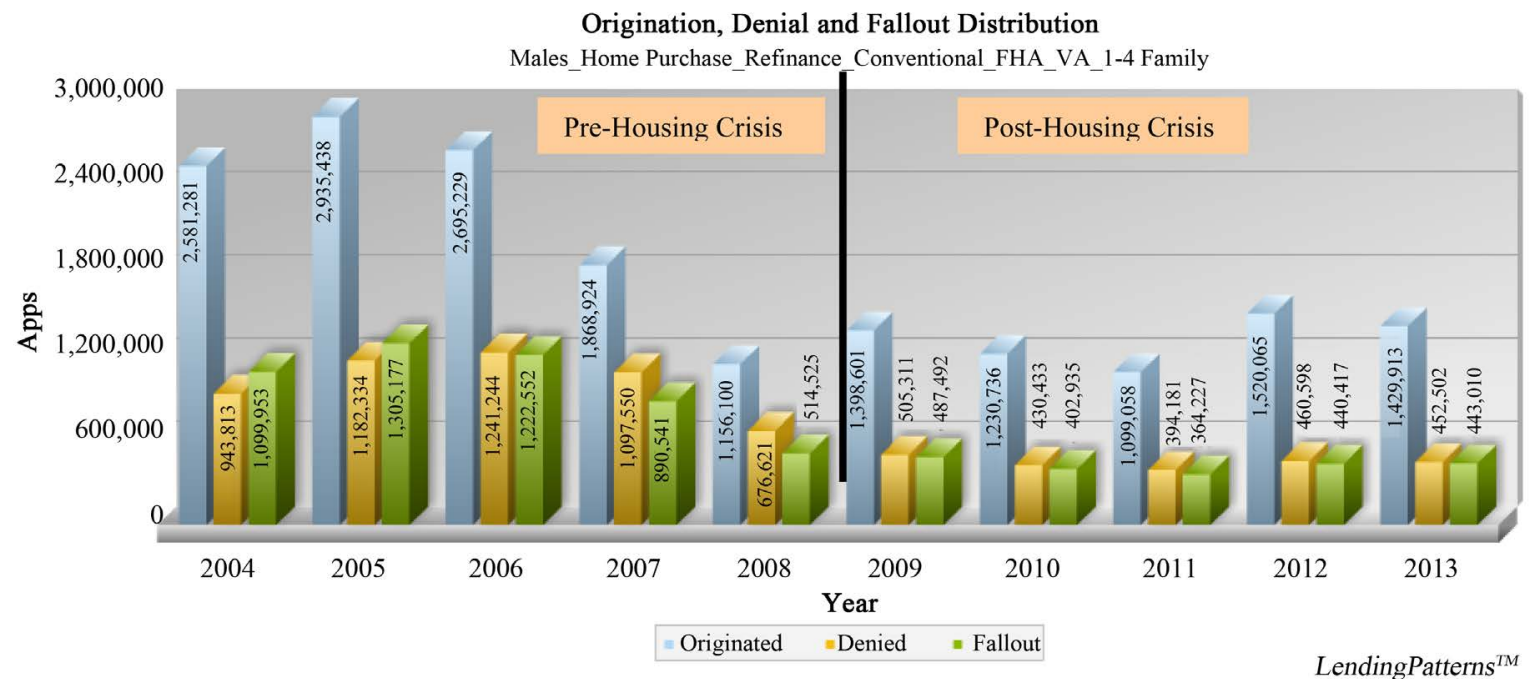

Figure 2. Female applicants: pre and post housing crisis origination, denial, fallout distribution.

\section{Results}

This section shows the results of the statistical procedures used to detect gender differences in origination, denial, and fallout rates. The three main procedures used were Independent t-test, One-way ANOVA, and One-Sample t-test. The independent t-tests were used to check for differences in loan processing rates for males and versus females across all races as well as within each race (e.g., white males vs. white females). The ANOVAs were used to assess for differences within gender by race (e.g., white females vs. Hispanic females). The one-sample-test is used as a Litmus test [21] to investigate differences in loan processing rates as compared to white males. The origination, denial, and fallout rates were determined to have a normal distribution after examining standardized skewness and employing the Shapiro-Wilks test.

\subsection{Independent t-Test: Origination, Denial and Fallout Rate}

To determine in the post housing-crisis era if there was a difference between male and female origination, denial and fallout rates, a set of independent-samples t-tests was used. A summary of the results are provided in Table 1 and are based on the homogeneity of variances. Origination rates for pre and post housing crisis were not 
Table 1. (a) Origination, denial, fallout rates by gender: Independent sample t-test. (b) Independent samples t-test by gender.

(a)

\begin{tabular}{|c|c|c|c|c|c|c|}
\hline & Rate & Gender & $\mathrm{N}$ & Mean & $\begin{array}{l}\text { Standard } \\
\text { deviation }\end{array}$ & $\begin{array}{c}\text { Standard error } \\
\text { mean }\end{array}$ \\
\hline \multirow{6}{*}{ Pre-housing crisis } & \multirow{2}{*}{ Origination } & Male & 25 & 45.97 & 7.55 & 1.51 \\
\hline & & Female & 25 & 47.29 & 7.52 & 1.50 \\
\hline & \multirow{2}{*}{ Denial } & Male & 25 & 28.22 & 7.68 & 1.54 \\
\hline & & Female & 25 & 28.14 & 7.55 & 1.51 \\
\hline & \multirow{2}{*}{ Fallout } & Male & 25 & 25.81 & 1.86 & 0.37 \\
\hline & & Female & 25 & 24.57 & 1.72 & 0.34 \\
\hline \multirow{6}{*}{ Post-housing crisis } & \multirow{2}{*}{ Origination } & Male & 25 & 53.47 & 6.46 & 1.29 \\
\hline & & Female & 25 & 54.50 & 6.21 & 1.24 \\
\hline & \multirow{2}{*}{ Denial } & Male & 25 & 24.30 & 5.01 & 1.00 \\
\hline & & Female & 25 & 24.39 & 4.69 & 0.94 \\
\hline & \multirow{2}{*}{ Fallout } & Male & 25 & 22.22 & 2.27 & 0.45 \\
\hline & & Female & 25 & 21.11 & 2.16 & 0.43 \\
\hline
\end{tabular}

(b)

\begin{tabular}{cccccccccc}
\hline & \multicolumn{2}{c}{ Levene's test for equal variances } & \multicolumn{5}{c}{ Independent samples test: t-test for equality means } \\
\cline { 2 - 9 } & Rate & $\mathrm{F}$ & $\mathrm{p}$ & $\mathrm{t}$ & $\mathrm{df}$ & Sig. (2-tailed) & Mean difference & $\mathrm{d}$ \\
\hline \multirow{3}{*}{$\begin{array}{c}\text { Pre-housing } \\
\text { crisis }\end{array}$} & Origination & 0.055 & 0.816 & -0.620 & 48 & 0.538 & -1.32 & 2.132 \\
& Denial & 0.021 & 0.884 & 0.038 & 48 & 0.970 & 0.08 & 2.153 \\
\hline \multirow{2}{*}{$\begin{array}{c}\text { Post-housing } \\
\text { crisis }\end{array}$} & Fallout & 0.004 & 0.953 & 2.443 & 48 & 0.018 & 0.567 & -1.03 & 1.793 \\
& Origination & 0.226 & 0.637 & -0.576 & 48 & 0.507 & 1.373 \\
& Denial & 0.300 & 0.587 & -0.059 & 48 & 0.954 & -0.08 & 1.11 & 0.626 \\
\hline
\end{tabular}

significantly different between male and female. Pre-housing crisis, origination rates were for males $(\mathrm{M}=45.97$, $\mathrm{SD}=7.55)$ and females $(\mathrm{M}=47.29, \mathrm{SD}=7.52), \mathrm{t}(48)=-0.620, \mathrm{p}=0.538, \mathrm{~d}=0.18$. Post-housing crisis, origination rates for males were $(\mathrm{M}=53.47, \mathrm{SD}=6.46)$ and females $(\mathrm{M}=54.50, \mathrm{SD}=6.21), \mathrm{t}(48)=-0.576, \mathrm{p}=$ $0.567, \mathrm{~d}=-0.01$. Denial rates for pre and post housing crisis were not significantly different between males and females. Pre-housing crisis denial rates were for males $(\mathrm{M}=28.22$, $\mathrm{SD}=7.68)$ and females $(\mathrm{M}=28.14$, $\mathrm{SD}=$ 7.55), $\mathrm{t}(48)=0.038, \mathrm{p}=0.970, \mathrm{~d}=-0.01$. Post-housing crisis denial rates were for males $(\mathrm{M}=24.30, \mathrm{SD}=5.01)$ and females $(\mathrm{M}=24.39, \mathrm{SD}=4.69), \mathrm{t}(48)=-0.059, \mathrm{p}=0.954, \mathrm{~d}=0.02$. Fallout rates for pre and post housing crisis were not significantly different between males and females. Pre-housing crisis, fallout rates were for males $(\mathrm{M}=25.81, \mathrm{SD}=1.86)$ and females $(\mathrm{M}=24.57, \mathrm{SD}=1.72), \mathrm{t}(48)=2.443, \mathrm{p}=0.018, \mathrm{~d}=-0.69$. Post-housing crisis, fallout rates were for males $(\mathrm{M}=22.2, \mathrm{SD}=2.27)$ and females $(\mathrm{M}=21.11, \mathrm{SD}=2.16), \mathrm{t}(48)=1.78, \mathrm{p}=$ $0.082, \mathrm{~d}=-0.50$.

Shown in Table 2 are the results from a set of independent samples t-tests, which was used to detect differences between male and female origination, denial and fallout rates after controlling for race. The normality assumption held for each group and homogeneity of variance assumption for all groups except Native Americans' denial rate. In the latter case, t-value for equal variances not assumed was reported in Table 2. In the posthousing crisis era, the results indicated no statistically significant difference between male and female for origination, denial and fallout rates, with some exceptions. These exceptions were for white male versus white female fallout rates and Asian male versus Asian female denial rates. The fallout rate for white male $(\mathrm{M}=20.28$, $\mathrm{SD}=0.74)$ was significantly higher than females $(\mathrm{M}=18.87, \mathrm{SD}=0.75, \mathrm{t}(8)=2.98, \mathrm{p}=0.018, \mathrm{~d}=-1.89)$. The 
denial rate for Asian males $(M=17.80, \mathrm{SD}=1.27)$ was significantly lower than females $(\mathrm{M}=19.64, \mathrm{SD}=1.12$, $\mathrm{t}(8)=-2.42, \mathrm{p}=0.042, \mathrm{~d}=-1.53)$.

\subsection{ANOVA Origination Rate}

The results presented in Table 3 capture the one-way ANOVA for the race effect on origination rate. The Levene's test revealed that the homogeneity variance assumption by race was violated for the female origination

Table 2. (a) Origination, denial and fallout rates: group statistics; (b) Independent samples t-test by gender, controlling for race.

(a)

\begin{tabular}{|c|c|c|c|c|c|c|}
\hline Race & Rate & Gender & $\mathrm{N}$ & Mean & Std. deviation & Std. error mean \\
\hline \multirow{6}{*}{ White } & \multirow{2}{*}{ Origination } & Male & 5 & 59.90 & 1.82 & 0.81 \\
\hline & & Female & 5 & 62.17 & 1.58 & 0.71 \\
\hline & \multirow{2}{*}{ Denial } & Male & 5 & 19.82 & 1.11 & 0.50 \\
\hline & & Female & 5 & 18.96 & 0.89 & 0.40 \\
\hline & \multirow{2}{*}{ Fallout } & Male & 5 & 20.28 & 0.74 & 0.33 \\
\hline & & Female & 5 & 18.87 & 0.75 & 0.34 \\
\hline \multirow{6}{*}{ Black } & \multirow{2}{*}{ Origination } & Male & 5 & 46.73 & 2.35 & 1.05 \\
\hline & & Female & 5 & 47.77 & 2.46 & 1.10 \\
\hline & \multirow{2}{*}{ Denial } & Male & 5 & 30.41 & 1.42 & 0.63 \\
\hline & & Female & 5 & 30.40 & 1.77 & 0.79 \\
\hline & \multirow{2}{*}{ Fallout } & Male & 5 & 22.86 & 0.99 & 0.44 \\
\hline & & Female & 5 & 21.84 & 0.85 & 0.38 \\
\hline \multirow{6}{*}{ Hispanic } & \multirow{2}{*}{ Origination } & Male & 5 & 52.09 & 2.87 & 1.28 \\
\hline & & Female & 5 & 53.01 & 2.89 & 1.29 \\
\hline & \multirow{2}{*}{ Denial } & Male & 5 & 25.66 & 1.74 & 0.78 \\
\hline & & Female & 5 & 25.71 & 1.68 & 0.75 \\
\hline & \multirow{2}{*}{ Fallout } & Male & 5 & 22.25 & 1.14 & 0.51 \\
\hline & & Female & 5 & 21.28 & 1.23 & 0.55 \\
\hline \multirow{6}{*}{ Asian } & \multirow{2}{*}{ Origination } & Male & 5 & 60.63 & 2.53 & 1.13 \\
\hline & & Female & 5 & 59.36 & 2.30 & 1.03 \\
\hline & \multirow{2}{*}{ Denial } & Male & 5 & 17.80 & 1.27 & 0.57 \\
\hline & & Female & 5 & 19.64 & 1.12 & 0.50 \\
\hline & \multirow{2}{*}{ Fallout } & Male & 5 & 21.57 & 1.30 & 0.58 \\
\hline & & Female & 5 & 21.00 & 1.22 & 0.55 \\
\hline \multirow{6}{*}{$\begin{array}{c}\text { Native } \\
\text { American }\end{array}$} & \multirow{2}{*}{ Origination } & Male & 5 & 48.01 & 3.87 & 1.73 \\
\hline & & Female & 5 & 50.21 & 4.88 & 2.18 \\
\hline & \multirow{2}{*}{ Denial } & Male & 5 & 27.83 & 0.68 & 0.31 \\
\hline & & Female & 5 & 27.22 & 1.32 & 0.59 \\
\hline & \multirow{2}{*}{ Fallout } & Male & 5 & 24.16 & 3.99 & 1.78 \\
\hline & & Female & 5 & 22.56 & 3.74 & 1.67 \\
\hline
\end{tabular}


(b)

\begin{tabular}{|c|c|c|c|c|c|c|c|c|}
\hline \multirow{2}{*}{ Race } & \multicolumn{3}{|c|}{ Levene’s test for equal variances } & \multicolumn{5}{|c|}{ Independent samples test: t-test for equality of means } \\
\hline & Rate & F & $\mathrm{p}$ & $\mathrm{t}$ & df & Sig. (2-tailed) & Mean difference & d \\
\hline \multirow{3}{*}{ White } & Origination & 0.45 & 0.519 & -2.11 & 8 & 0.068 & -2.27 & 1.33 \\
\hline & Denial & 0.87 & 0.377 & 1.36 & 8 & 0.212 & 0.86 & -0.86 \\
\hline & Fallout & 0.05 & 0.831 & 2.98 & 8 & 0.018 & 1.41 & -1.89 \\
\hline \multirow{3}{*}{ Black } & Origination & 0.02 & 0.887 & -0.68 & 8 & 0.514 & -1.04 & 0.43 \\
\hline & Denial & 1.35 & 0.279 & 0.02 & 8 & 0.986 & 0.02 & -0.01 \\
\hline & Fallout & 0.17 & 0.694 & 1.75 & 8 & 0.118 & 1.02 & -1.11 \\
\hline \multirow{3}{*}{ Hispanic } & Origination & 0.01 & 0.924 & -0.50 & 8 & 0.627 & -0.92 & 0.32 \\
\hline & Denial & 0.03 & 0.866 & -0.04 & 8 & 0.966 & -0.05 & 0.03 \\
\hline & Fallout & 0.00 & 0.988 & 1.29 & 8 & 0.233 & 0.97 & -0.82 \\
\hline \multirow{3}{*}{ Asian } & Origination & 0.00 & 0.978 & 0.83 & 8 & 0.433 & 1.26 & -0.52 \\
\hline & Denial & 0.02 & 0.890 & -2.42 & 8 & 0.042 & -1.84 & 1.53 \\
\hline & Fallout & 0.00 & 0.990 & 0.72 & 8 & 0.495 & 0.57 & -0.45 \\
\hline \multirow{3}{*}{$\begin{array}{c}\text { Native } \\
\text { American }\end{array}$} & Origination & 0.94 & 0.361 & -0.79 & 8 & 0.453 & -2.20 & 0.50 \\
\hline & Denial & 9.03 & 0.017 & 0.90 & 6 & 0.400 & 0.60 & -0.57 \\
\hline & Fallout & 0.14 & 0.714 & 0.65 & 8 & 0.532 & 1.60 & -0.41 \\
\hline
\end{tabular}

rates $(\mathrm{p}=0.025)$. As such, the Welch's $\mathrm{F}$ test and the posthoc James-Howell procedures were used. For females, the ANOVA revealed a statistically significant main race effect on origination rates, Welch's $F(4,9.87)=30.07$, $\mathrm{p}<0.001, \omega^{2}=0.83$. Omega squared has shown approximately $83 \%$ of the total variation, in the female's origination rate, was attributable to differences between the borrowers' race. Comparisons using Games-Howell posthoc procedure indicated that origination rate for white females $(\mathrm{M}=62.17, \mathrm{SD}=1.58)$ was significantly higher than Black females $(M=47.77, S D=2.46, p<0.001, d=6.27)$, Hispanic females $(M=53.01, S D=2.89$, $\mathrm{p}=0.004, \mathrm{~d}=3.25)$, Native American females $(\mathrm{M}=50.21, \mathrm{SD}=4.88, \mathrm{p}=0.019, \mathrm{~d}=3.30)$ but not for Asian females $(M=59.36, S D=2.30, p=0.264, d=1.42)$. For males, the Levene's $F$ test revealed that the homogeneity variance assumption by race was met for the male origination rates $(\mathrm{p}=0.182)$. The ANOVA showed a statistically significant race effect on origination rates, $\mathrm{F}(4,20)=27.62, \mathrm{p}<0.001, \eta^{2}=0.84$. Eta squared indicated that approximately $84 \%$ of the total variation, in the male's origination rates, was attributable to differences between the borrower's race. Comparisons using the Turkey posthoc procedure showed that origination rate for white males $(M=59.90, S D=1.82)$ was significantly higher than Black males $(M=46.73, S D=2.35, p<0.001$, $\mathrm{d}=6.27)$, Hispanic males $(\mathrm{M}=52.09, \mathrm{SD}=2.87, \mathrm{p}=0.009, \mathrm{~d}=3.25)$, Native American males $(\mathrm{M}=48.01, \mathrm{SD}=$ 3.87, $\mathrm{p}=0.006, \mathrm{~d}=3.93)$ but not for Asian males $(\mathrm{M}=60.63, \mathrm{SD}=2.53, \mathrm{p}=0.982, \mathrm{~d}=-0.33)$.

\subsection{ANOVA Denial Rate}

The one-way ANOVA results for the race effect on denial rate are presented in Table 4. The Levene's test revealed that the homogeneity variance assumption for denial rates by race was met for the female $(p=0.305)$ and the male $(\mathrm{p}=0.277$ For females, the ANOVA revealed a statistically significant race effect on denial rates, F(4, $9.87)=67.71, \mathrm{p}<0.001, \eta^{2}=0.93$. Eta squared has shown approximately $93 \%$ of the total variation in the female's denial rate was attributable to differences between the borrower's race. Comparisons using the Tukey posthoc procedure indicated that the denial rate for white females $(\mathrm{M}=19.82$, $\mathrm{SD}=1.11)$ was significantly lower than Black females $(\mathrm{M}=30.40, \mathrm{SD}=1.77, \mathrm{p}<0.001, \mathrm{~d}=-8.15)$, Hispanic females $(\mathrm{M}=25.71, \mathrm{SD}=$ 1.68, $\mathrm{p}<0.001, \mathrm{~d}=-5.03)$, Native American females $(\mathrm{M}=27.22, \mathrm{SD}=1.32, \mathrm{p}<0.001, \mathrm{~d}=-7.34)$ but not for Asian females $(\mathrm{M}=19.64, \mathrm{SD}=1.12, \mathrm{p}=0.936, \mathrm{~d}=-0.67)$. For males, the ANOVA revealed a statistically 
Table 3. (a) One-way ANOVA: post-housing crisis origination rates by race and statistics; (b) ANOVA; (c) Comparisons dependent variable $=$ origination rate, Posthoc tests $=$ Games-Howell.

(a)

\begin{tabular}{ccccc}
\hline & \multicolumn{3}{c}{ Test of homogeneity of variances } & Sig. \\
\hline Gender & Levene's Statistic & df1 & df2 & 0.182 \\
Male & 1.732 & 4 & 20 & 0.025 \\
\hline
\end{tabular}

(b)

\begin{tabular}{|c|c|c|c|c|c|c|c|c|c|c|}
\hline Gender & & $\begin{array}{l}\text { Sum of } \\
\text { squares }\end{array}$ & $\mathrm{df}$ & $\begin{array}{l}\text { Mean } \\
\text { square }\end{array}$ & $\mathrm{F}$ & Sig. & Welch's F ${ }^{\mathrm{a}}$ & df1 & df2 & Sig. \\
\hline \multirow{3}{*}{ Male } & Between groups & 41.88 & 4 & 10.47 & 27.62 & 0.000 & 5.71 & 4 & 9.72 & 0.012 \\
\hline & Within groups & 81.65 & 20 & 4.08 & & & & & & \\
\hline & Total & 123.53 & 24 & & & & & & & \\
\hline \multirow{3}{*}{ Female } & Between groups & 38.49 & 4 & 9.62 & 20.16 & 0.000 & 8.37 & 4 & 9.74 & 0.003 \\
\hline & Within groups & 73.26 & 20 & 3.66 & & & & & & \\
\hline & Total & 111.75 & 24 & & & & & & & \\
\hline
\end{tabular}

(c)

\begin{tabular}{|c|c|c|c|c|c|c|}
\hline Gender & (I) Race & (J) Race & Mean difference ${ }^{\mathrm{b}}$ (I-J) & Std. error & Sig. & $\mathrm{d}$ \\
\hline \multirow{4}{*}{ Male } & \multirow{4}{*}{ White } & Black & 13.17 & 1.33 & 0.000 & 6.27 \\
\hline & & Hispanic & 7.81 & 1.52 & 0.009 & 3.25 \\
\hline & & Asian & -0.73 & 1.39 & 0.982 & -0.33 \\
\hline & & Native American & 11.89 & 1.91 & 0.006 & 3.93 \\
\hline \multirow{4}{*}{ Female } & \multirow{4}{*}{ White } & Black & 14.40 & 1.31 & 0.000 & 6.97 \\
\hline & & Hispanic & 9.16 & 1.47 & 0.004 & 3.93 \\
\hline & & Asian & 2.80 & 1.25 & 0.264 & 1.42 \\
\hline & & Native American & 11.95 & 2.30 & 0.019 & 3.30 \\
\hline
\end{tabular}

${ }^{\mathrm{a}}$ Asymptotically F distributed. ${ }^{\mathrm{b}}$ See Table 2 for mean values of origination rates.

significant race effect on denial rates, $\mathrm{F}(4,20)=85.24, \mathrm{p}<0.001, \eta^{2}=0.94$. Eta squared indicated approximately $94 \%$ of the total variation in the male's denial rate was attributable to differences between the borrower's race. Comparisons using the Tukey posthoc procedure showed that the denial rate for white males $(\mathrm{M}=$ 20.28, $\mathrm{SD}=0.74)$ was significantly lower than Black males $(\mathrm{M}=30.41, \mathrm{SD}=1.42, \mathrm{p}<0.001, \mathrm{~d}=-8.31)$, Hispanic males $(\mathrm{M}=25.66, \mathrm{SD}=1.74, \mathrm{p}<0.001, \mathrm{~d}=-4.01)$, Native American males $(\mathrm{M}=27.83, \mathrm{SD}=0.68, \mathrm{p}<$ $0.001, \mathrm{~d}=-8.68)$ but not for Asian males $(\mathrm{M}=17.80, \mathrm{SD}=1.27, \mathrm{p}=0.138, \mathrm{~d}=1.69)$.

\subsection{ANOVA Fallout Rate}

Shown in Table 5 is the one-way ANOVA results for the race effect on fallout rates. The Levene's test revealed that the homogeneity variance assumption by race was violated for the female $(\mathrm{p}<0.001)$ and the male $(\mathrm{p}<$ 0.001) fallout rates. As such, the Welch's F test and the posthoc Games-Howell procedures were employed. For females, the ANOVA revealed a statistically significant race effect on fallout rates, Welch's $F(4,9.74)=8.37, \mathrm{p}<$ $0.003, \omega^{2}=0.54$. Omega squared showed approximately $54 \%$ of the total variation in the female's fallout rate is attributable to differences between the borrower's race. Comparisons using Games-Howell posthoc procedure indicated that the fallout rate for white females $(\mathrm{M}=18.87$, $\mathrm{SD}=0.75)$ was significantly lower than Black females $(\mathrm{M}=21.84, \mathrm{SD}=0.85, \mathrm{p}<0.001, \mathrm{~d}=-3.71)$, Hispanic females $(\mathrm{M}=21.28, \mathrm{SD}=1.23, \mathrm{p}<0.001, \mathrm{~d}=$ $-2.37)$, Native American females $(\mathrm{M}=22.56, \mathrm{SD}=3.74, \mathrm{p}<0.001, \mathrm{~d}=-1.37)$, but it was not lower than Asian 
Table 4. (a) One-way ANOVA: post-housing crisis denial rates by race and gender; (b) ANOVA.

(a)

\begin{tabular}{ccccc}
\hline & \multicolumn{3}{c}{ Test of homogeneity of variances } & Sig. \\
\hline Gender & Levene's statistic & df1 & df2 & .305 \\
Male & 1.295 & 4 & 20 & .277 \\
\hline
\end{tabular}

(b)

\begin{tabular}{|c|c|c|c|c|c|c|}
\hline Gender & & Sum of squares & df & Mean square & $\mathrm{F}$ & Sig. \\
\hline \multirow{3}{*}{ Male } & Between groups & 569.54 & 4 & 142.39 & 85.24 & 0.000 \\
\hline & Within groups & 33.41 & 20 & 1.67 & & \\
\hline & Total & 602.95 & 24 & & & \\
\hline \multirow{3}{*}{ Female } & Between groups & 489.41 & 4 & 122.35 & 62.71 & 0.000 \\
\hline & Within groups & 39.02 & 20 & 1.95 & & \\
\hline & Total & 528.44 & 24 & & & \\
\hline $\begin{array}{c}\text { Comparisons dependent variable = } \\
\text { denial rate, Posthoc tests = } \\
\text { Tukey gender }\end{array}$ & (I) Race & (J) Race & $\begin{array}{c}\text { Mean } \\
\text { difference }^{\mathrm{b}}(\mathrm{I}-\mathrm{J})\end{array}$ & Std. error & Sig. & d \\
\hline \multirow{4}{*}{ Male } & \multirow{4}{*}{ White } & Black & -10.59 & 0.82 & 0.000 & -8.31 \\
\hline & & Hispanic & -5.84 & 0.82 & 0.000 & -4.01 \\
\hline & & Asian & 2.02 & 0.82 & 0.138 & 1.69 \\
\hline & & Native American & -8.00 & 0.82 & 0.000 & -8.68 \\
\hline \multirow{4}{*}{ Female } & \multirow{4}{*}{ White } & Black & -11.44 & 0.88 & 0.000 & -8.15 \\
\hline & & Hispanic & -6.75 & 0.88 & 0.000 & -5.03 \\
\hline & & Asian & -0.68 & 0.88 & 0.936 & -0.67 \\
\hline & & Native American & 11.95 & 2.30 & 0.019 & 3.30 \\
\hline
\end{tabular}

\footnotetext{
${ }^{\mathrm{b}}$ See Table 2 for mean values of denial rates.
}

females $(\mathrm{M}=21.00, \mathrm{SD}=1.22, \mathrm{p}=0.933, \mathrm{~d}=-2.103)$. For males, the ANOVA revealed a statistically significant race effect on fallout rates, Welch's $F(4,20)=5.71, p=0.012, \omega^{2}=0.43$. Omega squared indicated approximately $43 \%$ of the total variation in the male's fallout rate was attributable to differences between the borrower's race. Comparisons using the Turkey posthoc procedure showed that the fallout rate for white males $(\mathrm{M}=$ 20.28, $\mathrm{SD}=0.74)$ was significantly lower than Black males $(\mathrm{M}=22.86, \mathrm{SD}=0.99, \mathrm{p}<0.001, \mathrm{~d}=-2.95)$, Hispanic males $(\mathrm{M}=22.25, \mathrm{SD}=1.14, \mathrm{p}<0.001, \mathrm{~d}=-2.05)$, Native American males $(\mathrm{M}=24.16, \mathrm{SD}=3.99$, $\mathrm{p}<$ $0.001, \mathrm{~d}=-1.35)$ but not for Asian males $(\mathrm{M}=21.57, \mathrm{SD}=1.30, \mathrm{p}=0.138, \mathrm{~d}=-1.22)$.

\subsection{One-Sample t-Test Origination Rate}

To see if there was a difference in origination rates between white males and females by race as well as non white males, a set of one-sample t-tests were used. The test results are presented in Table 6. For females, the test indicated that the white male's origination rate (59.9) was significantly greater than origination rates for Black females $(\mathrm{M}=47.77, \mathrm{SD}=2.46, \mathrm{t}(4)=-11.04, \mathrm{p}<0.001, \mathrm{~d}=-2.21)$, Hispanic females $(\mathrm{M}=53.01, \mathrm{SD}=2.89$, $\mathrm{t}(4)=-5.34, \mathrm{p}=0.006, \mathrm{~d}=-1.070)$, and Native American females $(\mathrm{M}=50.21, \mathrm{SD}=4.88, \mathrm{t}(4)=-4.44, \mathrm{p}=$ 0.006, $d=-0.89)$, but it was not statistically different from Asian females $(M=59.36$, $S D=2.30, t(4)=-0.52$, $\mathrm{p}=0.315, \mathrm{~d}=-0.10)$. While the white male's origination rate was significantly less than the origination rate for 
Table 5. (a) One-way ANOVA: post-housing crisis fallout rates by race and gender; (b) ANOVA; (c) Comparisons dependent variable $=$ fallout rate, Posthoc tests $=$ Games-Howell.

(a)

\begin{tabular}{ccccc}
\hline & \multicolumn{3}{c|}{ Test of homogeneity of variances } & Sig. \\
\hline Gender & Levene's statistic & df1 & df2 & 0.000 \\
Male & 15.230 & 4 & 20 & 0.001 \\
\hline
\end{tabular}

(b)

\begin{tabular}{|c|c|c|c|c|c|c|c|c|c|c|}
\hline Gender & & $\begin{array}{l}\text { Sum of } \\
\text { squares }\end{array}$ & $\mathrm{df}$ & $\begin{array}{c}\text { Mean } \\
\text { square }\end{array}$ & $\mathrm{F}$ & Sig. & Welch’s F & df1 & df2 & Sig. \\
\hline \multirow{3}{*}{ Male } & Between groups & 41.88 & 4 & 10.47 & 465.15 & 0.000 & 5.71 & 4 & 10 & 0.012 \\
\hline & Within groups & 81.65 & 20 & 4.08 & & & & & & \\
\hline & Total & 123.53 & 24 & & & & & & & \\
\hline \multirow{3}{*}{ Female } & Between groups & 38.49 & 4 & 9.62 & 363.10 & 0.000 & 8.37 & 4 & 10 & 0.003 \\
\hline & Within groups & 73.26 & 20 & 3.66 & & & & & & \\
\hline & Total & 111.75 & 24 & & & & & & & \\
\hline
\end{tabular}

(c)

\begin{tabular}{|c|c|c|c|c|c|c|}
\hline Gender & (I) Race & (J) Race & Mean difference ${ }^{\mathrm{b}}(\mathrm{I}-\mathrm{J})$ & Std. error & Sig. & $\mathrm{d}$ \\
\hline \multirow{4}{*}{ Male } & \multirow{4}{*}{ White } & Black & 13.17 & 1.33 & 0.000 & 6.27 \\
\hline & & Hispanic & 7.81 & 1.52 & 0.009 & 3.25 \\
\hline & & Asian & -0.73 & 1.39 & 0.982 & -0.33 \\
\hline & & Native American & 11.89 & 1.91 & 0.006 & 3.93 \\
\hline \multirow{4}{*}{ Female } & \multirow{4}{*}{ White } & Black & 14.40 & 1.31 & 0.000 & 6.97 \\
\hline & & Hispanic & 9.16 & 1.47 & 0.004 & 3.93 \\
\hline & & Asian & 2.80 & 1.25 & 0.264 & 1.42 \\
\hline & & Native American & 11.95 & 2.30 & 0.019 & 3.30 \\
\hline
\end{tabular}

${ }^{\mathrm{a}}$ Asymptotically F distributed. ${ }^{\mathrm{b}}$ See Table 2 for mean values of fallout rates.

white females $(\mathrm{M}=62.17, \mathrm{SD}=1.58, \mathrm{t}(4)=3.21, \mathrm{p}=0.032, \mathrm{~d}=0.64)$. For males, the test indicated that the white male's origination rate was significantly greater than origination rates for Black males $(\mathrm{M}=46.73, \mathrm{SD}=$ 2.35, $\mathrm{t}(4)=-12.55, \mathrm{p}<0.001, \mathrm{~d}=-2.5)$, Hispanic males $(\mathrm{M}=52.09, \mathrm{SD}=2.87, \mathrm{t}(4)=-6.09, \mathrm{p}=0.004, \mathrm{~d}=$ -1.22), and for Native American males $(\mathrm{M}=48.01, \mathrm{SD}=3.87, \mathrm{t}(4)=-6.87, \mathrm{p}=0.002, \mathrm{~d}=-1.37)$, but it was not statistically different from Asian males $(\mathrm{M}=60.63, \mathrm{SD}=2.53, \mathrm{t}(4)=0.64, \mathrm{p}=0.566, \mathrm{~d}=0.13)$.

\subsection{One-Sample t-Test Denial Rate}

To determine if there was a difference in denial rates between white males and females by race as well as other non white males, a set of one-sample t-tests was used. Table 6 presents a comparison between white males to females by race and nonwhite males. For females, the test indicated that white male's (19.82) denial rate was significantly less than denial rates for Black females $(\mathrm{M}=30.40, \mathrm{SD}=1.77, \mathrm{t}(4)=13.33, \mathrm{p}<0.001, \mathrm{~d}=2.67)$, Hispanic females $(\mathrm{M}=25.71, \mathrm{SD}=1.68, \mathrm{t}(4)=7.84, \mathrm{p}=0.001, \mathrm{~d}=1.57)$, and for Native American females $(\mathrm{M}=$ 27.22, $\mathrm{SD}=1.32, \mathrm{t}(4)=12.53, \mathrm{p}<0.001, \mathrm{~d}=2.51)$, but it was not significantly different from Asian females $(\mathrm{M}=19.64, \mathrm{SD}=1.12, \mathrm{t}(4)=-0.36, \mathrm{p}=0.735, \mathrm{~d}=-0.07)$ and white females $(\mathrm{M}=18.96, \mathrm{SD}=0.89, \mathrm{t}(4)=$ $-2.17, \mathrm{p}=0.095, \mathrm{~d}=-0.43$ ). For males, the one-sample t-test indicated that the white male's denial rate was significantly less than denial rates for Black males $(\mathrm{M}=30.41, \mathrm{SD}=1.42, \mathrm{t}(4)=16.69, \mathrm{p}<0.001, \mathrm{~d}=3.34)$, Hispanic males $(\mathrm{M}=25.66, \mathrm{SD}=1.74, \mathrm{t}(4)=7.52, \mathrm{p}=0.002, \mathrm{~d}=1.50)$, Native American males $(\mathrm{M}=27.83$, 
Table 6. One-sample t-test: white males compared to females and minority males.

\begin{tabular}{|c|c|c|c|c|c|c|}
\hline \multirow[b]{2}{*}{ Race $^{\mathrm{a}}$} & \multirow[b]{2}{*}{ Gender } & \multicolumn{5}{|c|}{ White male origination rate $=59.900$} \\
\hline & & $\mathrm{t}$ & df & Sig. & Mean difference & d \\
\hline \multirow{2}{*}{ White } & Male & 0.00 & 4 & & & \\
\hline & Female & 3.21 & 4 & 0.032 & 2.27 & 0.64 \\
\hline \multirow{2}{*}{ Black } & Male & -12.55 & 4 & 0.000 & -13.17 & -2.51 \\
\hline & Female & -11.04 & 4 & 0.000 & -12.13 & -2.21 \\
\hline \multirow{2}{*}{ Hispanic } & Male & -6.09 & 4 & 0.004 & -7.81 & -1.22 \\
\hline & Female & -5.34 & 4 & 0.006 & -6.89 & -1.07 \\
\hline \multirow{2}{*}{ Asian } & Male & 0.64 & 4 & 0.556 & 0.73 & 0.13 \\
\hline & Female & -0.52 & 4 & 0.630 & -0.54 & -0.10 \\
\hline \multirow{2}{*}{$\begin{array}{c}\text { Native } \\
\text { American }\end{array}$} & Male & -6.87 & 4 & 0.002 & -11.89 & -1.37 \\
\hline & Female & -4.44 & 4 & 0.006 & -9.69 & -0.89 \\
\hline & & \multicolumn{5}{|c|}{ White male denial rate $=19.822$} \\
\hline Race $^{\mathrm{a}}$ & Gender & $\mathrm{t}$ & df & Sig. & Mean difference & $\mathrm{d}$ \\
\hline \multirow{2}{*}{ White } & Male & 0.00 & 4 & & & \\
\hline & Female & -2.17 & 4 & 0.095 & -0.86 & -0.43 \\
\hline \multirow{2}{*}{ Black } & Male & 16.69 & 4 & 0.000 & 10.59 & 3.34 \\
\hline & Female & 13.33 & 4 & 0.000 & 10.57 & 2.67 \\
\hline \multirow{2}{*}{ Hispanic } & Male & 7.52 & 4 & 0.002 & 5.84 & 1.50 \\
\hline & Female & 7.84 & 4 & 0.001 & 5.88 & 1.57 \\
\hline \multirow{2}{*}{ Asian } & Male & -3.54 & 4 & 0.024 & -2.02 & -0.71 \\
\hline & Female & -0.36 & 4 & 0.735 & -0.18 & -0.07 \\
\hline \multirow{2}{*}{$\begin{array}{c}\text { Native } \\
\text { American }\end{array}$} & Male & 26.18 & 4 & 0.000 & 8.00 & 5.24 \\
\hline & Female & 12.53 & 4 & 0.000 & 7.40 & 2.51 \\
\hline & & \multicolumn{5}{|c|}{ White male fallout rate $=\mathbf{2 0 . 2 7 6}$} \\
\hline Race $^{\text {a }}$ & Gender & $\mathrm{t}$ & df & Sig. & Mean difference & $\mathrm{d}$ \\
\hline \multirow{2}{*}{ White } & Male & 0.00 & 4 & & & \\
\hline & Female & -4.19 & 4 & 0.014 & -1.41 & -0.84 \\
\hline \multirow{2}{*}{ Black } & Male & 5.85 & 4 & 0.004 & 2.58 & 1.17 \\
\hline & Female & 4.12 & 4 & 0.015 & 1.56 & 0.82 \\
\hline \multirow{2}{*}{ Hispanic } & Male & 3.86 & 4 & 0.018 & 1.97 & 0.77 \\
\hline & Female & 1.82 & 4 & 0.143 & 1.00 & 0.36 \\
\hline \multirow{2}{*}{ Asian } & Male & 2.21 & 4 & 0.091 & 1.29 & 0.44 \\
\hline & Female & 1.32 & 4 & 0.258 & 0.72 & 0.26 \\
\hline \multirow{2}{*}{$\begin{array}{c}\text { Native } \\
\text { American }\end{array}$} & Male & 2.18 & 4 & 0.095 & 3.88 & 0.44 \\
\hline & Female & 1.37 & 4 & 0.244 & 2.29 & 0.27 \\
\hline
\end{tabular}

${ }^{\mathrm{a}} \mathrm{See}$ Table 2 for mean and standard deviations by race for origination, denial and fallout rates.

$\mathrm{SD}=0.68, \mathrm{t}(4)=12.53, \mathrm{p}<0.001, \mathrm{~d}=2.51)$, but it was significantly higher than Asian males $(\mathrm{M}=17.80, \mathrm{SD}=$ 1.27, $\mathrm{t}(4)=-3.54, \mathrm{p}=0.024, \mathrm{~d}=-0.71)$. 


\subsection{One-Sample t-Test Fallout Rate}

To determine if there was a difference in fallout rates between white males and females by race as well as other nonwhite males, a set of one-sample t-tests were used. Table 6 presents a comparison of white males to females by race and nonwhite males. For females, the test indicated that the white male's (20.28) fallout rate was significantly lower than fallout rates for Black females $(\mathrm{M}=21.84, \mathrm{SD}=0.85, \mathrm{t}(4)=4.12, \mathrm{p}=0.015, \mathrm{~d}=0.82)$ and significantly greater white females $(M=18.87, S D=0.075, t(4)=-4.19, p=0.014, d=-0.84)$, but it was not significantly different from Hispanic females $(\mathrm{M}=21.28, \mathrm{SD}=1.23, \mathrm{t}(4)=1.82, \mathrm{p}=0.143, \mathrm{~d}=0.36$ ), Native American females $(\mathrm{M}=22.56, \mathrm{SD}=3.74, \mathrm{t}(4)=1.37, \mathrm{p}=0.244, \mathrm{~d}=0.27)$, and Asian females $(\mathrm{M}=21, \mathrm{SD}=$ $1.22, \mathrm{t}(4)=1.32, \mathrm{p}=0.258, \mathrm{~d}=0.26$ ). For males, the test indicated that the white male's fallout rate was significantly less than fallout rates for Black males $(\mathrm{M}=22.86, \mathrm{SD}=0.99, \mathrm{t}(4)=5.85, \mathrm{p}=0.004, \mathrm{~d}=1.17)$, Hispanic males $(\mathrm{M}=22.25, \mathrm{SD}=1.14, \mathrm{t}(4)=3.86, \mathrm{p}=0.018, \mathrm{~d}=0.77)$, but it was not significantly different from Native American males $(\mathrm{M}=24.16, \mathrm{SD}=3.99, \mathrm{t}(4)=2.18, \mathrm{p}=0.095, \mathrm{~d}=0.44)$ and Asian males $(\mathrm{M}=21.57, \mathrm{SD}=1.30$, $\mathrm{t}(4)=2.21, \mathrm{p}=0.091, \mathrm{~d}=0.44)$.

\section{Conclusions}

The aim of this study was to examine the women's mortgage credit experience after the 2008 housing crisis by testing for statistical differences in origination, denial and fallout rates. These rates served as indicators capturing the performance of lenders' behavior during the loan underwriting process. Equally as important, the rates were used to shed light on the variation of the mortgage credit experience between men and women. On the positive side, the between-gender comparisons yield that the women's mortgage credit experience was statistically equal to men as measured by the experience origination, denial and fallout rates. These findings are consistent with other mortgage gender studies such as Dietrich and Johannsson [14], Robinson [8], and Awoonor-Williams [13]. Conversely, the findings are in contrast with some studies, Woodstock Institute [9] and Cyr [10]. Dietrich and Johannsson's [14] study used an underwriting decision model, controlled for economic factors and found 15 of 18 fair lending exams had no statistical gender effect on the decision to deny a mortgage. The Robinson [8] study found that applications for low-income women were more likely to be originated than men with similar income. Awoonor-Williams [13] found that being a female statistically did not increase the applicant's denial experience. Controlling for income and loan type, Diabate [12] found little differences between male and female mortgage originations. Hedderly [6] found no mortgage lending disparities against females, after controlling for MSA and loan type. In contrast, the Cyr [10] study found a higher denial rates for women. Woodstock's [9] study, also, found gender disparities in denial and origination rates across conventional and government-backed mortgages. Although their findings may be true, the samples were delimited to unique groups, such as pregnant women and six countries in the Chicago area.

The good news is that origination rates were up by (8\%) for men and (7\%) for women. Denial rates were down for men and women by (4\%). Fallout rates were down by (4\%) for men and (3\%) for women. The bad news is that the total number of mortgage originations was down. Pre-housing crisis, the annual-average mortgage originations for men and women were 3,045,770 and 2,247,394, respectively. Post-housing crisis, the annual-average mortgage originations for men and women, were 1,888,227 and 1,335,675 respectively. This trend implies tight mortgage lending restrictions has decreased opportunities for all applicants. The diameter of the mortgage pie has declined; thus, making it harder to enter the mortgage market compared to the pre-housing crisis era.

Within-gender comparison by applicants' race, white females had a statistically significant higher origination rate and lower denial rate than Black, Hispanic, and Native American females except Asian females, which was not significantly different. Further, the effect size had a high practical significance by Cohen's d scale. In other words, white females are more likely 1.30, 1.17, and 1.24 times to get a mortgage originated than Black, Hispanic and Native American females, respectively. Black, Hispanic and Native American females are 1.60, 1.36, and 1.44 times, respectively, more likely than white females denied a mortgage. White females had a statistically significant lower fallout rate than Black and Hispanic females with a high practical significance, but it was not significantly different from Asian and Native American females. Black and Hispanic females were 1.16 and 1.13 times, respectively, more likely to experience loan fallout during the underwriting process than white females. The Mohanty [11] study within gender comparison had similar results and found that the white females had a higher probability of home loan approvals than Asian and Hispanic females using a probit model. 
Within-race comparison by applicant's gender, there was no significant statistical difference between male and female in origination, denial and fallout rates with two exceptions. These exceptions included white men having a higher fallout rate than white women and Asian men having a lower denial rate than Asian women. The gender effect sizes had high practical significance. White male's mortgage applications or loans get classified as fallouts 1.08 times more likely than white females. Asian females get denied 1.10 times more likely than Asian The Litmus test comparison, white males compared to all females and non-white males. For the female's Litmus test, white males had a statistically significant higher origination rate than Black, Hispanic, and Native American females except Asian females, which was not significantly different. Further, the effect size had a high practical significance. White males were 1.26, 1.13, and 1.20 times more likely than Black, Hispanic and Native American females, respectively, to get a mortgage originated. The denial rate for white males was significantly lower than Black, Hispanic, and Native American females except Asian females, which was not significantly different. Further, the effect size had a high practical significance. Black, Hispanic, and Native American females were $1.54,1.30$, and 1.38 , respectively, more likely denied a mortgage than white males. The origination rate for white males was statistically lower than the white females with moderate, practical significances. White females were 1.04 times more likely to get a loan originated than white males. The independent t-test with the one-tailed probability value reinforces this finding. There was no significant difference between the white male denial rate and white female's.

For the male's Litmus test, white males had a statistically significant higher origination rate than Black, Hispanic, and Native American males except Asian males, which was not significantly different. White males were more likely 1.28, 1.15, and 1.25 times more likely than Black, Hispanic and Native American males, respectively, to get a mortgage originated. The denial rate for white males was significantly lower than Black, Hispanic, and Native American males. The effect size had a moderate to high practical significance. Black, Hispanic and Native American males were 1.54, 1.30, and 1.38, respectively, more likely denied a mortgage than white males. The denial rate for white males was significantly higher than Asian males with high, practical significance. White males were 1.12 times more likely than Asian males denied a loan. The fallout rate for white males was significantly lower than Black and Hispanic, but it was not significantly different from Asian and Native American males. The effect size had a moderate to high practical significance. Black and Hispanic males were 1.13 and 1.10 , respectively, more likely to have fallouts than white males.

In conclusion, at the aggregate, the results showed no statistical gender effect on origination, denial or fallout rates in the post-housing crisis era. Within-race, this relationship held up, except for white females who had a lower fallout rate than white males and Asian females who had a higher denial rate than Asian males. Withingender, controlling for applicants' race, white females had higher origination and lower denial rates than Black, Hispanic, and Native Americans but were not statistically different from Asian females. Comparing white males to females by applicants' race, the results indicated that $53 \%$ of the time white males had higher origination, lower denial, or lower fallout rates than females. While $40 \%$ of the time, the white male's mortgage experience was not statistically different from females. Seven percent of the time the white male's origination rate was lower than white females. In the final analysis, at no time did minority women have a better mortgage experience than white men. Merely 33\% of the time, minority women did experience no statistical difference than white men. Given these points, the applicant's gender had less of an effect on the mortgage credit experience than the applicant's race.

\section{References}

[1] White House (2015) Economic Report of the President. https://www.whitehouse.gov/sites/default/files/docs/cea_2015_erp.pdf

[2] Federal Home Loan Mortgage Corporation (2015) MiMi: Multi-Indicator Market Index. http://www.freddiemac.com/mimi/

[3] Federal National Mortgage Association (2015) National Housing Survey ${ }^{\mathrm{TM}}$. http://www.fanniemae.com/portal/research-and-analysis/mortgage-lender-survey.html

[4] Federal National Mortgage Association (2015) Mortgage Lender Sentiment Survey. http://www.fanniemae.com/portal/research-and-analysis/mortgage-lender-survey.html

[5] Hedderly, D.J. (2007) Women Take over: A Study of Gender and Homeownership. MBA Thesis, Pepperdine University, Malibu. http://search.proquest.com/docview/304707401?accountid=11490 
[6] National Council of Negro Women (2009) Assessing the Double Burden: Examining Racial and Gender Disparities in Mortgage Lending. http://ncnw.org/images/double_burden.pdf

[7] O’Connor, S.A. (1996) The Impact of Gender in the Mortgage Credit Market. Ph.D. Dissertations, University of Wisconsin, Milwaukee. http://search.proquest.com/docview/304298422?accountid=11490

[8] Robinson, J.K. (2001) A Home of One’s Own: Overcoming Gender and Familial Status Barriers to Homeownership. Ph.D. Dissertations, University of Massachusetts Amherst, Amherst. http://search.proquest.com/docview/230490541?accountid=11490

[9] Woodstock Institute (2015) Her Longer Road Home: Disparities in Mortgage Lending to Women in the Chicago Region. Woodstock Institute, Chicago. http://www.woodstockinst.org/research/her-longer-road-home-disparities-mortgage-lending-women-chicago-region

[10] Cyr, M.R. (2011) Gender, Maternity Leave, and Home Financing: A Critical Analysis of Mortgage Lending Discrimination against Pregnant Women. University of Pennsylvania Journal of Law and Social Change, 15, 109-141. http://scholarship.law.upenn.edu/jlasc/vol15/iss1/6

[11] Mohanty, L. (2001) Access to Housing Credit: A Study of Minority Women. PhD, Dissertations, University of California, Riverdale. http://search.proquest.com/docview/304692055?accountid=11490

[12] Diabate, A. (2000) The Question of Gender Discrimination in Mortgage Lending: A Cross-Regional Analysis. PhD Dissertations, The University of Tennessee, Knoxville. http://search.proquest.com/docview/ 304619830?accountid=11490

[13] Awoonor-Williams, P. (2004) Gender and Credit: An Analysis of Women's Experience in the Credit Market. PhD Dissertations, Howard University, Washington DC. http://search.proquest.com/docview/ 305196845?accountid=11490

[14] Dietrich, J. and Johannsson, H. (2005) Searching for Age and Gender Discrimination in Mortgage Lending. OCC Economics Working Paper 2005-2, Office of the Comptroller of the Currency. http://www.occ.gov/publications/publications-by-type/occ-working-papers/2008-2000/wp2005-2.pdf

[15] Jourdain-Earl, M. (2011) Foreclosure Crisis and Racial Disparities in Access to Mortgage Credit. http://compliancetech.com/wp-content/uploads/2013/11/The-Foreclosure-Crisis-and-Racial-Disparities-in-Access-to-M ortgage-Credit-2004-2009-_2-9-2011.pdf

[16] Dymski, G., Hernandez, J. and Mohanty, L. (2013) Race, Gender, Power, and the US Subprime Mortgage and Foreclosure Crisis: A Meso Analysis. Feminist Economics, 19, 124-151. http://dx.doi.org/10.1080/13545701.2013.791401

[17] Cheng, P., Lin, Z. and Liu, Y. (2009) Do Women Pay More for Mortgages? http://papers.ssrn.com/sol3/papers.cfm?abstract id=1491729

[18] Phillips, S. (2012) The Subprime Mortgage Calamity and the African American Woman. The Review Black Political Economy, 39, 227-237. http://dx.doi.org/10.1007/s12114-011-9107-1

[19] Baker, A.C. (2014) Eroding of Women: Gender and Subprime Foreclosure Crisis. Social Service Review, 88, 59-91. http://dx.doi.org/10.1086/675391

[20] Lending Patterns (2015) https://www.lendingpatterns.com.

[21] Federal Deposit Insurance Corporation (1996) Side by Side: A Guide to Fair Lending. https://www.fdic.gov/regulations/resources/side/side.pdf 